\title{
Avaluadors anònims de PAPERS i estadística d'articles de l'any 2011
}

Avaluadors anònims de l'any 2011

Aguiar, Fernando (IESA/CSIC)

Aierdi Urraza, Xabier (EHU)

Albert Guardiola, Maria (UA)

Albertos Puebla, Juan Miguel (UV)

Almeda, Elisabet (UB)

Ayuso Sánchez, Luis (UMA)

Baer, Alejandro (UCM)

Ballart Hernández, Francesc (UAB)

Bazo Royo, María Teresa (EHU)

Bestard Camps, Juan (UB)

Blanco, Ismael (IGOP/UAB)

Boso Gaspar, Alejandro (UPF)

Bravo Moreno, Ana (UGR)

Caínzos López, Miguel Ángel (USC)

Calvo Borovia, Kerman (USAL)

Calvo García, Manuel (UNIZAR)

Camarero Rioja, Luis (UNED)

Capdevila Gómez, Arantxa (URV)

Carabaña, Julio (UCM)

Carrasquer Oto, Pilar (UAB)

Casas Aznar, Ferran (UDG)

Cea d'Ancona, María Ángeles (UCM)

Cebolla Boada, Héctor (UNED-CEACS/ FJM)

Coller Porta, Xavier (YALE)

Collet-Sabé, Jordi (UVIC)

Cordero, Julia (CCHS/CSIC)

Cortina Trilla, Clara (CCHS/CSIC)
Criado, María (UCM)

Domingo, Andreu (CED/UAB)

Domínguez Pérez, Marta (UCM)

Escardíbul, Josep-Oriol (UB)

Espluga, Josep Lluís (UAB)

Espina Montero, Álvaro (UCM-MEH)

Espuny Tomás, Maria Jesús (UAB)

Esteve, Albert (UAB)

Estruch Gibert, Joan (UAB)

Fernández Enguita, Mariano (UCM)

Ferrer Julià, Ferran (UAB)

Flaquer, Lluís (UAB)

Flecha García, José Ramón (UB)

Gaitán Muñoz, Lourdes (UCM)

Galais González, Carolina (UAB)

Ganuza, Ernesto (IESA/CSIC)

García Calavia, Miguel Ángel (UV)

García Sanz, Benjamín (UCM)

García Castaño, Francisco Javier (UGR)

Garrido, Luis (UNED)

Garrido Fernández, Fernando E. (IESA/ CSIC)

González Enríquez, Carmen (UNED)

González Fernández, Manuel (UPO)

González Ferrer, Amparo (CCHS/CSIC)

González López, María (UPF)

Graces Mascareñas, Blanca (UPF)

Guiu Payà, Jordi (UPF) 
Gutiérrez Palacios, Rodolfo (UNIOVI) Hernández Corrochano, David (CCHS/ CSIC)

Hernández Corrochano, Elena (UNED)

Herranz Aguayo, Inmaculada (UCLM)

Herranz de Rafael, Gonzalo (UAL)

Herranz González, Roberto (USC)

Hita Alonso, Cecilia (UGR)

Izquierdo Benito, María Jesús (UAB)

Izquierdo Brichs, Ferran (UAB)

Jurado Guerrero, Teresa (UNED)

Laespada, María Teresa (Deusto)

León, Ana (Exeter)

León Medina, Francisco José (UDG)

López Sala, Ana (CCHS/CSIC)

López Sintas, Jordi (UAB)

Malo Ocaña, Miguel (USAL)

Mantecón, Alejandro (UA)

Marbán, Vicente (UAH)

Mari-Klose, Margarita (UB)

Mari Klose, Pau (CCHS/CSIC-UB)

Marqués Perales, Ildefonso (US-CEA)

Martínez Buján, Raquel (UDC)

Martínez Sanmartí, Roger (UOC)

Mayoral Arqué, Dolors (UDL)

Merino Pareja, Rafael (UAB)

Miguel Quesada, Francisco José (UAB)

Mijares Molina, Laura (UCM)

Miller Moya, Luis Miguel (EHU)

Montagut Antolí, Teresa (UB)

Morata Tierra, Francesc (UAB)

Moreno Colom, Sara (UAB)

Moreno Márquez, Gorka (EHU)

Moyano Estrada, Eduardo (IESA/CSIC)

Navarro Yáñez, Clemente (UPO)

Ortega Gaspar, Marta (UMA)

Parella Rubio, Sònia (UAB)
Pastor Gosálbez, Inmaculada (URV)

Pastor Seller, Enrique (UM)

Pérez Beltrán, Carmelo (UGR)

Planas, Jordi (UAB)

Prieto Rodríguez, Carlos (UCM)

Puigvert Mallart, Lídia (UB)

Pujades Rúbies, Isabel (UB)

Radl Philip, Rita (USC)

Rambla, Xavier (UAB)

Ramió Matas, Carles (UPF)

Riba Romeva, Clara (UPF)

Rinken, Sebastián (IESA/CSIC)

Rodríguez García, Dan (UAB)

Rodríguez Pascual, Iván (UHU)

Sáez Giol, Lluís (UAB)

Sanchís, Enric (UV)

Sarasa Urdiola, Sebastià (UPF)

Sarrible Pedroni, Graciela (UB)

Serradell Pumareda, Olga (UAB)

Serrano del Rosal, Rafael (IESA/CSIC)

Setién, María (Deusto)

Soler Masó, Pere (UDG)

Sordé Martí, Teresa (UAB)

Soriano-Miras, Rosa (UGR)

Suárez, Liliana (UAM)

Tarabini, Aina (UAB)

Tena Sánchez, Jordi (CNRS-Sorbonne)

Tezanos Tortajada, José Félix (UNED)

Tobío Soler, Constanza (UC3M)

Torrent, Joan (UOC)

Troiano Gomà, Helena (UAB)

Val, Consuelo del (UNED)

Vallejo Peña, Francisco (UMA)

Vancea, Mihaela (UPF)

Verd Pericàs, Joan Miquel (UAB)

Vicente Torrado, Trinidad (Deusto) 
Avaluadors segons el sexe

\begin{tabular}{lcc}
\hline & Nre. & $\%$ \\
\hline Homes & 75 & 59,52 \\
Dones & 51 & 40,47 \\
Total & 126 & 100,00 \\
\hline
\end{tabular}

Avaluadors segons l'afiliació institucional

\begin{tabular}{lrr}
\hline & Nre. & $\%$ \\
\hline Avaluadors de la UAB & 26 & 20,63 \\
Avaluadors externs a la UAB & 100 & 79,37 \\
Total & 126 & 100,00 \\
\hline
\end{tabular}

Estadística d'articles de l'any 2011

Articles rebuts

\begin{tabular}{lrr}
\hline & Nre. & $\%$ \\
\hline Amb decisió editorial presa & 82 & 83,67 \\
En avaluació & 16 & 16,33 \\
Retirats per l'autor & 0 & 0,00 \\
Total & 98 & 100,00 \\
\hline
\end{tabular}

Articles avaluats

\begin{tabular}{lrr}
\hline & Nre. & $\%$ \\
\hline No publicables & 41 & 50,00 \\
Reavaluables & 18 & 21,95 \\
Publicables amb modificacions & 16 & 19,51 \\
Publicables sense modificacions & 7 & 8,54 \\
Total & 82 & 100,00 \\
\hline
\end{tabular}

